\title{
Serotonin-Depleted Rats are Capable of Learning in Active Place Avoidance, a Spatial Task Requiring Cognitive Coordination
}

\author{
T. PETRÁSEK, A. STUCHLÍK \\ Institute of Physiology, Academy of Sciences of the Czech Republic, Prague, Czech Republic
}

Received December 9, 2008

Accepted January 8, 2009

\begin{abstract}
Summary
Neurotransmitter substrate of spatial cognition belongs to current topics in behavioral neuroscience. The present study examined the effects of serotonin depletion with p-chlorophenylalanine on learning of rats in active place avoidance, a spatial task requiring allothetic mapping and cognitive coordination and highly dependent upon hippocampus. Serotonin depletion transiently increased locomotor activity in response to footshocks, but it did not change the avoidance efficiency measured by three spatial parameters. These results suggest that serotonin neurotransmission is not crucial for cognitive coordination and allothetic learning, i.e. the processes, which are crucial for active place avoidance performance.
\end{abstract}

\section{Key words}

Spatial cognition - Cognitive coordination • Learning • Place avoidance $\bullet$ Serotonin depletion

\section{Corresponding author}

A. Stuchlík, Institute of Physiology ASCR, Vídeňská 1083, 14220 Prague, Czech Republic. Fax. +420241062488 . E-mail: stuchlik@biomed.cas.cz

Serotonin or 5-hydroxytryptamine (5-HT) is an indolamine neurotransmitter which exerts many physiological functions in the central nervous system (CNS). The functions regulated by serotonin range from feeding and motor regulation to emotion and aggression (Smythies 2005). The vast majority of serotonergic pathways originate in the brainstem nuclei, known as raphe nuclei, from which axons arise to innervate virtually whole CNS (Stanford 2001). The raphe nuclei can be roughly divided to the caudal nuclei projecting to brainstem and spinal cord, dorsal nuclei, which innervate the cerebral cortex, striatum, thalamus and median raphe nuclei providing innervation to hippocampal formation, septum, and neocortical regions, namely frontal, occipital, parietal and cingulated cortices (Stanford 2001, Smythies 2005). Serotonin is synthesized from tryptophan by tryptophan hydroxylase producing 5-hydroxytryptophan, which is decarboxylated to serotonin. Its synaptic actions are mediated by several classes of receptors $\left(5-\mathrm{HT}_{1-7}\right]$ which are further divided into subgroups. Action of serotonin is terminated by its reuptake from synapses via 5-HT transporter (Povlock and Amara 1997).

The involvement of serotonin neurotransmission in cognition is a matter of intense debate. Although 5-HT has clear effects on cognition in humans (McEntee and Crook 1991) and degeneration of serotonergic (and cholinergic) fibers is found in Alzheimer's disease (Yamamoto and Hirano 1985), results from the animal studies are less conclusive. Blocking of serotonin synthesis did not induce a deficit in the Morris water maze (Richter-Levin and Segal 1989) and reduction of 5-HT levels by 5,7-dihydroxytryptamine (5-DHT) did not also affect spatial learning (Riekkinen et al. 1990). Contrarily, there is evidence that multiple 5-HT receptor subtypes may interact to regulate animal learning. It is often suggested that serotonin modulates cognitive processes especially by the interaction with cholinergic systems (Zarrindast 2006).

Ten years ago, a cognitive task called active place avoidance was designed in our laboratory (Bureš et al. 1998). In this task, rats are trained to actively explore a slowly rotating arena and avoid an unmarked sector, 
which is defined as a stable position in the room. Animals must thus localize the shock sector and walk in the safe part of the arena in a direction opposite to arena rotation. It has been shown that in place avoidance tasks, rats use both the intramaze cues (urine, droppings, and scent marks) as well as visual landmarks located in the room. However, both these classes of information are conflicted by arena rotation in this task. Therefore, the animals must segregate spatial cues into representations of the arena and room (Kubík et al. 2006) and select the room frame as the only relevant coordinate system for navigation. This process was described as "cognitive coordination" (Wesierska et al. 2005). The requirement to separate cues into coherent subsets makes the task useful for studying involvement of receptors in the cognition (Stuchlík et al. 2008). Since the active place avoidance task involves higher cognitive functions and is highly dependent upon hippocampus, which receives 5-HT projections from median raphe nucleus, the present study aimed at testing the hypothesis that brain serotonin depletion via systemic injection of $p$-chlorophenylalanine will interfere with efficient learning in this task.

Animal procedures were in accordance with the Animal Protection Code of the Czech Republic and with EU directive 86/609/EEC. Twenty-four male Long-Evans rats (4-5 months, 300-450 g), obtained from the Institute's breeding colony, were housed in pairs in $30 \times 30 \times 40 \mathrm{~cm}$ cages in an air-conditioned vivarium with a stable temperature $\left(22^{\circ} \mathrm{C}\right)$ and $12 / 12$ light/dark cycle. Rats were gently implanted with a hypodermic needle, piercing the rat's skin between its shoulders, and creating a small loop on the needle with tweezers. The loop provided purchase for an alligator clip connecting a shock-delivering cable. Water and food was freely available.

Serotonin depletion was pursued by systemic administration of $p$-chlorophenylalanine ( $p$ CPA; SigmaAldrich, CR). $p$ CPA was suspended in the $0.5 \%$ solution of gum arabic in saline $(0.9 \% \mathrm{NaCl})$ and administered in two single (500 mg/kg, i.p.) injections dispensed on each of two consecutive days (injected at 9:00). Total volume of each injection was $4 \mathrm{ml} / \mathrm{kg}$. Behavioral training started $72 \mathrm{~h}$ after the second injection. We chose this protocol because it was demonstrated to consistently reduce 5-HT levels in brain by $91 \%$ (Dringenberg et al. 1995, Dyer and Cain 2007). All groups consisted of eight animals. Two control groups were used; one untreated (intact controls) and one injected with gum arabic only (shaminjected).

The apparatus was described in detail elsewhere
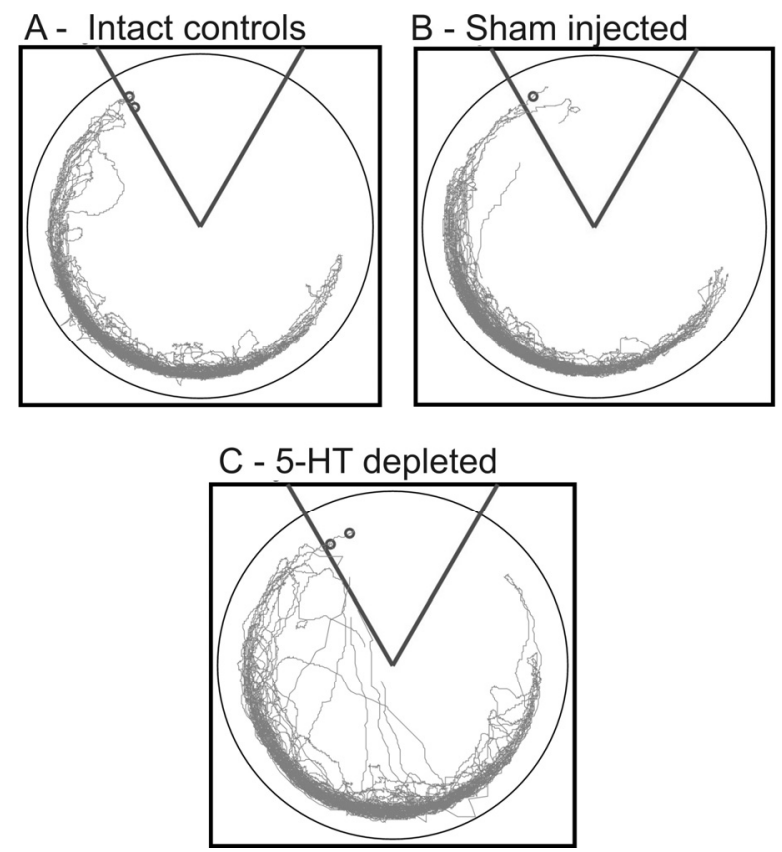

Fig. 1. Examples of typical trajectories of rats from the final day of active place avoidance training. Panel A shows the track of animal from intact controls, Panel B demonstrates a track of rat from sham-injected group and Panel C illustrates a track from 5-HT depleted rat. All animals efficiently avoided the punished zone. Small circles denote footshocks delivered upon entrance into the shock sector.

(Bureš et al. 1998, Stuchlík et al. 2008). Briefly, it was a circular elevated arena located in a room containing many visual cues. Every animal was placed onto the arena, which rotated clockwise at one rotation per minute. The animals were to avoid an unmarked, $60^{\circ}$ sector defined by its relationships to room cues. A tracking system (iTrack, Biosignal, USA) recorded the rat's position every $40 \mathrm{~ms}$. Coordinates were stored for off-line analysis (TrackAnalysis; Biosignal, USA). Whenever the rat entered the to-be-avoided sector, the tracking system delivered a mild electric shock ( $50 \mathrm{~Hz}, 0.5 \mathrm{~s}, 0.2-0.8 \mathrm{~mA})$ and counted an entrance. Shocks were delivered through the implanted needle and the grounded arena floor. The current was gradually increased in the beginning of the first session, starting at $0.2 \mathrm{~mA}$, until rapid escape reaction was elicited. Vast majority animals responded appropriately to $0.4 \mathrm{~mA}$, there were also no systematic differences in the threshold current between groups. Four daily sessions of training in active place avoidance task were conducted, each lasting $20 \mathrm{~min}$, and pursued between 9:00 and 12:00.

The following parameters were analyzed. The total distance walked per session reflected the locomotor activity. Two measures of within-session spatial 

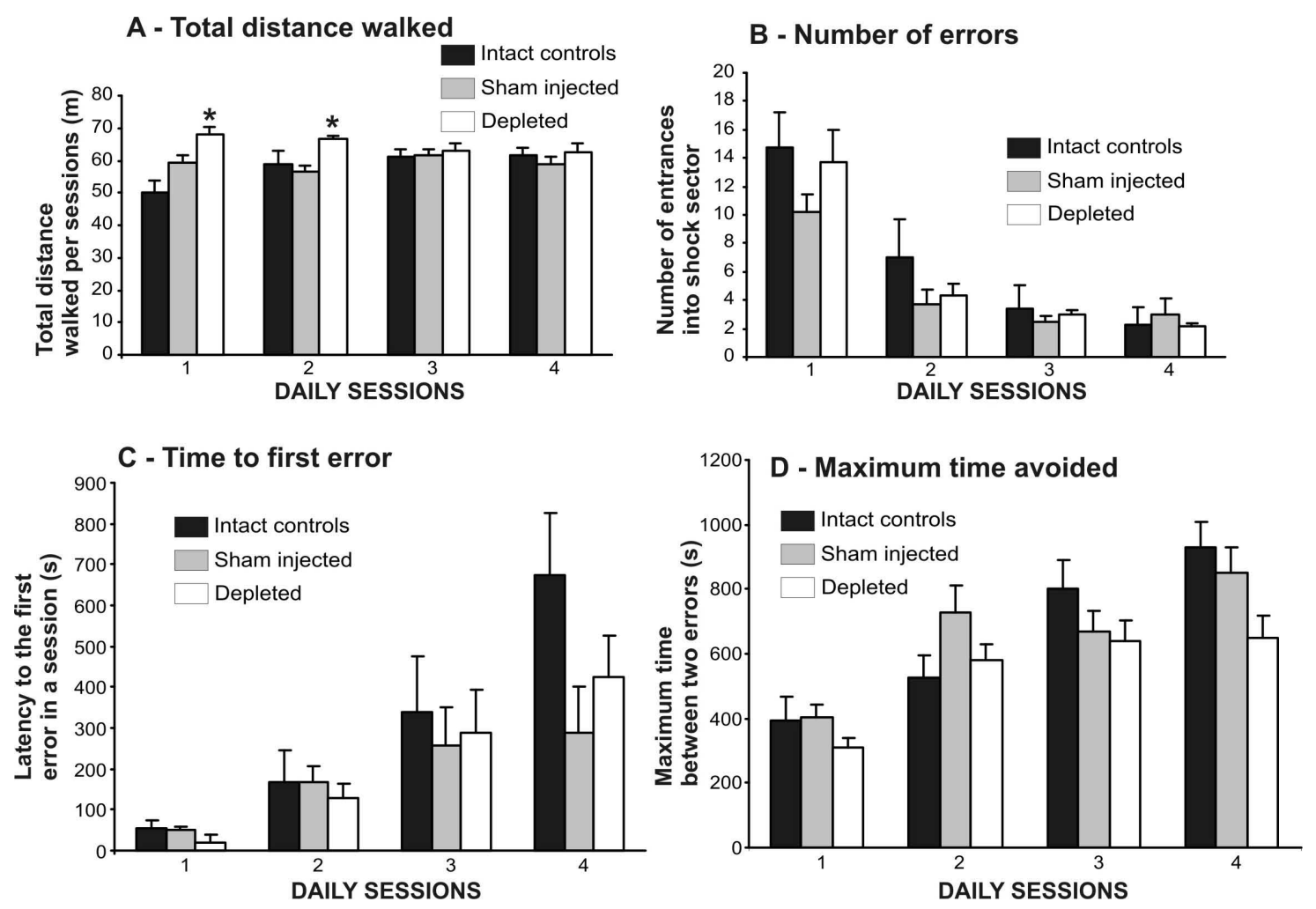

Fig. 2. Panel A reveals the effect of 5-HT depletion on locomotor activity measured as total distance (mean \pm S.E.M.). 5-HT depleted rats exhibited higher total distances in the beginning of the training, on the last two days of training, all groups walked similar distance. $* \mathrm{P}<0.05$. Panel B shows no effect of 5-HT depletion on the number of entrances (mean \pm S.E.M.), a consistent training-induced improvement is seen in all groups. Panel C illustrates the effect of serotonin depletion on the time to first error (mean \pm S.E.M.), a measure to between-session learning; no significant between-group differences were found. A two-way ANOVA and a test of linear trend showed that this parameter increased with training $(P<0.05)$, however, no differences were found between groups $(P>0.05)$. Panel D demonstrates no effect of 5-HT depletion on the maximum time avoided (mean \pm S.E.M.); another measure of within-session learning.

performance were the number of errors (number of entrances into the shock sector) and maximum time between two errors (maximum time avoided). Latency to the first error was used as a measure of between-session learning. The data were analyzed with a two-way ANOVA (groups $\mathrm{x}$ sessions) with repeated measures on sessions and with the Tukey's HSD post-hoc test (Statistica 7, StatSoft, $\mathrm{CR})$. Significance was accepted at $\mathrm{P}<0.05$.

Visual observation of control rats and animals treated with $p$ CPA did not reveal any signs of stress during and after injections; all basic behaviors were retained. Animals responded to footshocks with mild startles and rapid escapes, suggesting that the perception of shocks was retained despite serotonin depletion. Examples of typical tracks of rats from all three groups are shown in Figure 1.

First, we analyzed the locomotor activity. The quantification of total distance indicated that there was a difference between groups (Fig. 2A). A two-way ANOVA revealed a significant effect of groups (F (2.21)
$=3.95 ; \mathrm{P}<0.05)$, but no effect of sessions $(\mathrm{F}(3.63)=$ $0.83 ; \mathrm{P}>0.05)$. A significant interaction between the two factors was found $(\mathrm{F}(6.63)=3.80 ; \mathrm{P}<0.05)$. A post-hoc analysis of groups revealed that animals depleted of 5-HT walked slightly but significantly longer distance than sham-injected animals $(\mathrm{P}<0.05)$. Post-hoc analysis of the interaction showed that difference between depleted and control rats was caused by significant difference in the first two sessions $(\mathrm{P}<0.05)$, however, in sessions $3-4$, the trend of increased locomotion in depleted rats did not reach statistical significance $(\mathrm{P}=0.054)$.

Analysis of the number of errors revealed no between-group differences, but the performance was improving with training in all groups (Fig. 2B). A twoway ANOVA failed to show a significant main effect of groups $(\mathrm{F}(2.21)=0.7 ; \mathrm{P}>0.05)$ as well as no interaction $(\mathrm{F}(6.63)=4.07 ; \mathrm{P}>0.05)$, but it revealed a significant main effect of sessions (F (3.63) $=44.21 ; \mathrm{P}<0.05)$. Posthoc analysis of sessions showed that performance was better in sessions 2-4 than in session 1, suggesting 
consistent training-induced improvement in all groups.

Measuring the time to first entrance again revealed the improvement during training, but no between-group differences (Fig. 2C). A two-way ANOVA revealed no effect of groups $(\mathrm{F}(2.21)=1.01$; $\mathrm{P}>0.05$ ), but it found a main effect of sessions ( $\mathrm{F}$ (3.63) $=13.98 ; \mathrm{P}<0.05)$. No interaction was found $(\mathrm{F}(6.63)=$ 1.21 ; $\mathrm{P}>0.05)$. Post-hoc analysis of the factor of sessions revealed a consistent learning-induced improvement in this parameter. Finally, analysis of the maximum time avoided again showed no between-group differences (Fig. 2D). A two-way ANOVA failed to reveal a main effect of groups $(\mathrm{F}(2.21)=1.72 ; \mathrm{P}>0.05)$, no interaction $(\mathrm{F}(6.63)=2.16 ; \mathrm{P}>0.05)$, but it revealed a significant main effect of sessions $(\mathrm{F}(3.63)=28.89$; $\mathrm{P}<0.05)$. Post-hoc analysis again revealed that performance measured by this parameter was improving with training in all groups.

Results of the present study show that serotonin depletion induced by the application of 5-HT biosynthesis inhibitor $p$ CPA did not change the spatial efficiency in the active place avoidance task. No alterations in avoidance were measured by any of the spatially sensitive parameters, including the retrievalsensitive measure of the latency to the first entrance in a session (Wesierska et al. 2005). The only change that we detected was a slight increase in locomotor activity after $p$ CPA treatment, prominent mainly in the beginning of the training.

Regarding locomotor activity, however, it is usually described in the literature that a decrease in the locomotion is observed after serotonin depletion. For example, Dringenberg et al. (1995) described reduction of spontaneous exploratory activity after $p \mathrm{CPA}$ treatment in the open-field test. Importantly, this study reported no overt sensorimotor impairments after the same dose of $p C P A$ as used in the present study. This result is in agreement with our present results, since depleted animals were capable of readily learning the task. Our study detected a transient significant increase in locomotion, which seems to contradict the study of Dringenberg et al. (1995), but there are also reports of increased locomotion after $p \mathrm{CPA}$ treatment and/or median raphe nuclei lesion (Köhler and Lorens 1978). Since the 5-HT may increase the reaction of animals to stress (Tanke et al. 2008), we propose that transient hyperlocomotion in the present study (most prominent in the initial session) represents an alteration of the locomotion in response to negative reinforcement (footshocks).
Regarding the absence of effect of 5-HT depletion on the spatial learning in this study, our results are in accordance with several other studies. For instance, Beiko et al. (1997) demonstrated no effect of the $p$ CPA application (in the same regimen and doses as in this study) on learning of rats in the water maze. Similar results were obtained by Dyer and Cain (2007), who found no impairment of water maze learning after pCPA as well. However, another study (Hritcu et al. 2007) found impairments in the working memory (WM), but not in the reference memory in the radial maze, after 5-DHT neurotoxic lesion of serotonergic neurons in the rat. Although WM is a function partially involved in the efficient performance of the active place avoidance task (Stuchlík et al. 2008), the main aspect of the task is reference memory (Bureš et al. 1998), because the shock sector location does not change between sessions. Therefore, the overall performance of 5-HT-depleted rats could remain the same as in controls. Moreover, another study (Cassaday et al. 2003) showed that 5-HT depletion may interfere only with acquisition of rules of WM task, but not with the pure WM. In light of their results, it is conceivable that in the place avoidance task, animal learned the rules of the task in the initial sessions and performance on the following days thus remained unaffected. One of the few memory tasks being significantly impaired by 5 -HT depletion is object-recognition paradigm (Lieben et al. 2004); however, there was apparent dissociation between the effects of depletion on the object recognition vs. spatial learning, showing no influence upon the latter (Lieben et al. 2004).

To summarize, the present study shows no effect of serotonin depletion upon cognitive functions measured in the place avoidance task, which involves a process of cognitive coordination, and these result support the concept that serotonergic transmission, despite being clearly involved in human learning, is not crucial for spatial cognition in rodents, including spatial mapping and cognitive coordination.

\section{Conflict of Interest}

There is no conflict of interest.

\section{Acknowledgements}

The study was supported by GACR grants 309/07/0341 and 309/09/0286 and by AV0Z50110509. We thank P. Luketic for language revision and J. Vorlíček for statistical assistance. 


\section{References}

BEIKO J, CANDUSSO L, CAIN DP: The effect of nonspatial water maze pretraining in rats subjected to serotonin depletion and muscarinic receptor antagonism: a detailed behavioural assessment of spatial performance. Behav Brain Res 88: 201-211, 1997.

BUREŠ J, FENTON AA, KAMINSKY Y, WESIERSKA M, ZAHÁLKA A: Rodent navigation after dissociation of the allocentric and idiothetic representations of space. Neuropharmacology 37: 689-699, 1998.

CASSADAY HJ, NORMAN C, SHILLIAM CS, VINCENT C, MARSDEN CA: Intraventricular 5,7-dihydroxytryptamine lesions disrupt acquisition of working memory task rules but not performance once learned. Prog Neuropsychopharmacol Biol Psychiatry 27: 147-156, 2003.

DRINGENBERG HC, HARGREAVES EL, BAKER GB, COOLEY RK, VANDERWOLF. CH: p-chlorophenylalanine-induced serotonin depletion: reduction in exploratory locomotion but no obvious sensory-motor deficits. Behav Brain Res 68: 229-237, 1995.

DYER K, CAIN DP: Water maze impairments after combined depletion of somatostatin and serotonin in the rat. Behav Brain Res 181: 85-95, 2007.

HRITCU L, CLICINSCHI M, NABESHIMA T: Brain serotonin depletion impairs short-term memory, but not longterm memory in rats. Physiol Behav 91: 652-657, 2007.

KÖHLER C, LORENS SA: Open field activity and avoidance behavior following serotonin depletion: a comparison of the effects of parachlorophenylalanine and electrolytic midbrain raphe lesions. Pharmacol Biochem Behav 8: $223-233,1978$.

KUBÍK S, STUCHLÍK A, FENTON AA: Evidence for hippocampal role in place avoidance other than merely memory storage. Physiol Res 55: 445-452, 2006.

LIEBEN CK, VAN OORSOUW K, DEUTZ NE, BLOKLAND A: Acute tryptophan depletion induced by a gelatinbased mixture impairs object memory but not affective behavior and spatial learning in the rat. Behav Brain Res 151: 53-64, 2004.

MCENTEE WJ, CROOK TH: Serotonin, memory and the aging brain. Psychopharmacol (Berl) 103: 143-149, 1991.

POVLOCK SL, AMARA SG: The structure and function of noerepinephrine, dopamine and serotonin transporters. In: Neurotransmitter Transporters. MEA REITH (ed), Humana Press, New York, 1997, pp 1-28.

RICHTER-LEVIN G, SEGAL M: Spatial performance is severely impaired in rats with combined reduction of serotonergic and cholinergic transmission. Brain Res 477: 404-407, 1989.

RIEKKINEN P, SIRVIO J, RIEKKINEN P: Interaction between raphe dorsalis and nucleus basalis magnocellularis in spatial learning. Brain Res 527: 342-345, 1990.

SMYTHIES J: Section V. Serotonin system. Int Rev Neurobiol 64: 217-268, 2005.

STANFORD SC: 5-Hydroxytryptamine. In: Neurotransmitters, Drugs and Brain Function. R WEBSTER (ed), John Wiley \& Sons Ltd, Chichester, UK, 2001, pp 187-209.

STUCHLÍK A, PETRÁSEK T, VALEŠ K: Dopamine $\mathrm{D}_{2}$ receptors and alpha1-adrenoceptors synergistically modulate locomotion and behavior of rats in a place avoidance task. Behav Brain Res 189: 139-144, 2008.

TANKE MA, ALSERDA E, DOORNBOS B, VAN DER MOST PJ, GOEMAN K, POSTEMA F, KORF J: Low tryptophan diet increases stress-sensitivity, but does not affect habituation in rats. Neurochem Int 52: 272-281, 2008.

WESIERSKA M, DOCKERY A, FENTON AA: Beyond memory, navigation, and inhibition: behavioral evidence for hippocampus-dependent cognitive coordination in the rat. J Neurosci 25: 2413-2419, 2005.

YAMAMOTO T, HIRANO A: Nucleus raphe dorsalis in Alzheimer's disease: Neurofibrillary tangles and loss of large neurons. Ann Neurol 17: 573-577, 1985.

ZARRINDAST MR: Neurotransmitters and cognition. In: Neurotransmitter Interactions and Cognitive Function. ED LEVIN (ed), Birkhäuser Verlag, Switzerland, 2006, pp 5-39. 